\title{
Tumores adrenocorticais na criança: da abordagem clínica à avaliação molecular
}

\author{
Childhood adrenocortical tumors: from a \\ clinical to a molecular approach
}

Sonir R. R. Antonini' ', Leandro M. Colli², Leticia Ferro',

Livia Mermejo², Margaret de Castro²

\section{SUMÁRIO}

Tumores do córtex adrenal (TCA) são mais frequentes em crianças, mas podem ocorrer em qualquer faixa etária. São classificados como funcionantes, não funcionantes (predominam no adulto), e mistos. 0 diagnóstico é baseado na avaliação clínica, hormonal e exames de imagem. Em crianças, o método de escolha para diferenciar entre benigno ou maligno é a classificação baseada no estadiamento do tumor. Alguns marcadores moleculares merecem destaque: além de mutações inativadoras no gene supressor tumoral TP53, há evidências de envolvimento do IGF2 em 90\% deTAC malignos, e mutações no éxon 3 do gene CTNNB1 foram encontradas em $6 \%$ dos TAC pediátricos. Além disso, microRNAs podem atuar como reguladores negativos da expressão gênica e participar da tumorigênese adrenocortical. Métodos para análise da expressão gênica permitem identificarTCA com prognóstico bom ou ruim, e espera-se que esses estudos possam facilitar o desenvolvimento de drogas para tratar pacientes de acordo com as vias de sinalização específicas que estiverem alteradas. Arq Bras Endocrinol Metab. 2011;55(8):599-606

\section{Descritores}

Tumor do córtex adrenal; doenças da adrenal; fatores de crescimento; proteína supressora de tumor P53; biologia molecular

\section{SUMMARY}

Adrenocortical tumors (ACT) are more frequent during childhood, but they can appear at any age. ACTs can be classified in functioning, nonfunctioning (mainly observed in adults) and mixed. The diagnosis is based on clinical, biochemical findings and imaging. In children, in order to classify ACT as benign or malignant, tumor staging classification is recommended. Regarding molecular markers some studies should be taken into account: besides TP53 mutations, previous studies have also provided evidences of IGF2 involvement in $90 \%$ of the malignant ACT. Mutations altering exon 3 of CTNNB1 gene have been found in $6 \%$ of childhood ACTs. In addition, microRNAs can act as negative regulators of gene expression by targeting mRNA controlling cell growth, differentiation and apoptosis and have been implicated in adrenal tumorigenesis. High-throughput methods to analyze genome-wide expression have been developed over the last decade and identified a subset of tumors with good or poor prognosis. In

Correspondência para: Margaret de Castro Departamento de Medicina Interna, Faculdade de Medicina de Ribeirão Preto, Universidade de São Paulo

Av. Bandeirantes, 3900 14049-900 - Ribeirão Preto, SP, Brasil

castrom@fmrp.usp.br

Recebido em 7/Out/2011 Aceito em 16/Out/2011

\section{INTRODUÇÃO}

$\mathrm{O}$ $s$ tumores adrenocorticais (TAC) apresentam incidência bimodal, sendo mais comuns nos primeiros cinco anos de vida e entre a $4^{\text {a }}$ e $5^{\text {a }}$ décadas de vida. Em todas as faixas etárias esses tumores são mais frequentes no sexo feminino $(1,2)$. Sua incidência varia de acordo com a região geográfica, sendo dez a quinze vezes mais elevada no Sul e Sudeste do Brasil, onde ocorrem apro- 
ximadamente 3,4 casos por milhão de crianças menores de 14 anos por ano (1). A razão da incidência mais elevada no Brasil ainda não é totalmente conhecida, porém a presença de uma única mutação germinativa no gene TP53 encontrada na maioria das crianças afetadas parece estar associada a esse risco elevado $(3,4)$. Por outro lado, carcinomas adrenocorticais (CAC) são raros, agressivos e apresentam prognóstico desfavorável $(2,5,6)$.

A suspeita diagnóstica geralmente ocorre em decorrência do excesso hormonal e, eventualmente, massa abdominal palpável. Crianças e adolescentes apresentam-se, tipicamente, com sinais e sintomas de produção excessiva de andrógenos, com quadro de virilização, sendo este o distúrbio hormonal mais comum, ou de excesso de cortisol, com quadro de síndrome de Cushing, ou ainda com ambos, sendo considerados tumores mistos. Em adultos, a síndrome mais comumente associada é a síndrome de Cushing. Em pacientes mais idosos, a maioria dos TAC tende a ser não funcionante $(1,2,6)$.

O diagnóstico do TCA é baseado, portanto, nos achados clínicos, nas dosagens hormonais e nos exames de imagem. Virilização é secundária à secreção aumentada de andrógenos, incluindo sulfato de de-hidroepiandrosterona (S-DHEA); $\Delta 5$-androstenediol e $\Delta 4$-androstenediona, os quais são convertidos a testosterona e $5 \alpha$-di-hidrotestosterona. Também são encontradas alterações nos níveis de testosterona, 17-hidroxiprogesterona e androstenediona. O padrão de secreção hormonal pode predizer o potencial maligno da lesão, como altas concentrações de S-DHEA ou estradiol elevado em homens. Raramente TAC apresentam secreção de estrógeno ou aldosterona. As dosagens de cortisol plasmático, do cortisol urinário livre, aldosterona plasmática e atividade de renina plasmática também devem ser realizadas para o diagnóstico e a avaliação da funcionalidade do tumor (7). Recentemente, uma abordagem combinando dosagens urinárias de esteroides, com base em cromatografia gasosa/espectrometria de massa seguida por um estudo computacional de algoritmos que permite determinar comportamentos biológicos baseados em dados empíricos (machine learning), examinou o valor diagnóstico para a detecção de malignidade em amostras de urina de pacientes com adenoma e carcinoma de adrenal. O diagnóstico foi confirmado por seguimento clínico, alterações histológicas e presença de metástases. O perfil de esteroides revelou um padrão predominantemente imaturo de esteroidogênese em estágio inicial em ACC. Os autores concluíram que a metabolômica dos esteroides urinários é uma ferramenta de biomarcador sensível e específico para discriminar tumores adrenais benignos de malignos. Esse teste, sensível, específico, rápido, de baixo custo e de triagem diagnóstica, poderá no futuro, potencialmente, eliminar a necessidade de imagens frequentes no acompanhamento de pacientes com TAC (8).

Exames de imagem são frequentemente utilizados no diagnóstico de TCA, avaliação de metástases e recidivas. Ressonância nuclear magnética (RNM), tomografia computadorizada (TC) e, mais recentemente, a tomografia de emissão de pósitrons utilizando 18F-fluoeodeoxyglicose (PDG-PET) têm sido as mais utilizadas para distinguir entre tumores malignos e benignos, sendo este último exame altamente sensível (97\%) e específico (91\%) em diferenciar tumores adrenais benignos de malignos (9). TC fornece informações para discriminar entre benignidade e malignidade, como tamanho, homogeneidade, presença de calcificações, áreas de necrose e invasão local. Carcinomas tendem a ser maiores que $5 \mathrm{~cm}$, apresentam margens irregulares e captam o contraste de forma não homogênea, além de invadirem estruturas vizinhas. Adenomas, em geral, são menores que $3 \mathrm{~cm}$, com bordas regulares, e captam o contraste de forma homogênea (10). Um coeficiente de atenuação menor que 10 Unidades Hounsfield (HU) e menor que $40 \mathrm{HU}$ quinze minutos após contraste com washout acima de $60 \%$ é sugestivo de adenoma (11). A RNM auxilia na diferenciação entre lesões benignas e malignas por meio da intensidade do sinal: lesões com alto sinal em T2 são sugestivas de carcinoma e baixo sinal em T2 sugere adenoma. RNM também fornece informações sobre invasão tumoral para veia cava e veias renais e, eventualmente, identificação de trombos (12).

O tamanho e o peso do tumor são os critérios mais usados para discriminação entre adenomas e carcinomas na criança (13). Duas escalas de estadiamento tumoral têm sido utilizadas para a classificação dos TAC pediátricos, a Escala de Macfarlane modificado por Sullivan $(6,14)$, que utiliza tamanho tumoral $(<$ ou $>$ $5 \mathrm{~cm})$ e a Escala de Sandrini modificada, que utiliza volume tumoral $\left(<\mathrm{ou}>200 \mathrm{~cm}^{3}\right)(\mathrm{l})$. A distinção na criança entre adenomas e carcinomas pela classificação histopatológica é difícil. Os critérios da classificação de Weiss são baseados em 9 escores: alto índice mitótico, mitoses atípicas, alta atividade nuclear, baixa porcentagem de células limpas, necrose, arquitetura difusa do tumor, invasão capsular, invasão sinusoidal e invasão venosa. Consideram-se malignos aqueles tumores cuja 
soma dos escores for maior que 3 (15). No entanto, essa classificação tem sido mais eficaz para a predição de pior prognóstico somente em TAC em adultos. Em crianças, o estadiamento tumoral é mais confiável para a predição de prognóstico (16).

A cirurgia é o tratamento mais efetivo para TAC, sendo a ressecção completa do tumor a única terapêutica que pode curar ou prolongar a sobrevida de forma significante, particularmente se a doença for detectada em estágios iniciais (I e II). Apesar da ressecção tumoral completa, a grande maioria dos pacientes com estágio III terá recidiva e doença metastática cinco anos após a ressecção tumoral; a sobrevida em cinco anos é de 30\% para o estágio III e 15\%-25\% para o estágio IV (17). A utilização da cirurgia laparoscópica para a ressecção dos TAC é controversa. Em decorrência do risco de ruptura da cápsula, da fragmentação tumoral e das dificuldades técnicas para retirar completamente o tumor com margens livres, o emprego dessa técnica deve ser restrito a pacientes adultos para remoção de TAC pequenos e possivelmente benignos (estágios I e II) (2). Em TAC pediátricos, a cirurgia por laparotomia é a via de escolha (6). Carcinomas aderentes ou invadindo estruturas adjacentes requerem excisão em bloco de rim, baço, hepatectomia ou pancreatectomia parcial, sendo que linfadenectomia deve também ser considerada. A reoperação deve ser considerada em casos de recorrência local ou metástases isoladas. Os pacientes aparentemente curados cirurgicamente requerem acompanhamento rigoroso e contínuo de longo prazo (18).

O mitotane (o-p'DDD), um agente adrenotóxico, é considerado a principal droga no tratamento dos TAC. Em estudo com adultos, $75 \%$ dos pacientes submetidos ao tratamento com mitotane tiveram a secreção hormonal controlada e aproximadamente $8 \%$ tiveram regressão do tumor. No entanto, mostrou-se ineficiente em prolongar a sobrevida dos pacientes com carcinomas de estágios III e IV (19). Além do tratamento monoterápico com mitotane, pacientes com doença metastática avançada podem se beneficiar de quimioterapia combinada com mitomicina, composta por etoposídeo, doxorrubicina e cisplatina ou com estreptozotocina $(20,21)$.

Alguns ensaios clínicos com terapia direcionada ao sistema IGF vêm sendo desenvolvidos, com base nos achados de diversos estudos que demonstraram que a hiper-regulação da via IGF é um dos principais eventos no desenvolvimento de CAC (22-25). O estudo envolvendo terapia direcionada ao sistema IGF já está em fase III e os pacientes portadores de CAC já foram recrutados para o uso do inibidor oral de pequenas moléculas do IGF-IR (NCI Trial No NCT00924989), com final do estudo previsto para 2013 (26). Um trabalho recente sugere que a falta de atividade antineoplásia de muitas drogas testadas pode ser devida ao aumento do metabolismo do CYP3A4 induzido pelo mitotane (27).

\section{BASES MOLECULARES DOS TUMORES ADRENOCORTICAIS}

O processo de tumorigênese adrenal ainda não está esclarecido, entretanto, nos últimos anos, diversos estudos têm auxiliado parcialmente na elucidação dos fenômenos moleculares envolvidos (3,4,28-31). A análise do modelo de inativação do cromossomo X em heterozigose em células somáticas femininas demonstrou que carcinomas derivam de células monoclonais enquanto adenomas têm origem tanto monoclonal quanto policlonal (32). Por meio de técnicas de hibridização genômica comparativa (HGC), alterações com ganho nos cromossomos 4, 5, 12, 19 e perdas em lp, 17p, 22p, $1 \mathrm{q}, 2 \mathrm{q}$ e $1 \mathrm{lq}$ foram identificadas em $61 \%$ dos adenomas e em $62 \%$ dos carcinomas (33). Estudos usando marcadores de microssatélites identificaram perda da heterozigose $(\mathrm{LOH})$ em carcinomas nas regiões $1 \mathrm{lql} 3$ (>90\%), 17pl3 (>85\%) e 2pl6 (92\%) $(28,34)$.

TAC podem se manifestar esporadicamente ou como parte de síndromes genéticas. A neoplasia endócrina múltipla tipo l é uma síndrome autossômica dominante causada por alteração no gene $M E N 1$, localizado no cromossomo 1lq13, que codifica uma proteína supressora tumoral. A função anormal dessa proteína predispõe a tumores de paratireoide, hipófise, ilhotas pancreáticas e TCA (35). LOH na região 1 lql3 ocorre em $20 \%$ dos TCA esporádicos, sendo essa alteração mais encontrada em carcinomas do que em adenomas; porém nenhuma mutação no gene MENl foi encontrada em TCA esporádicos estudados (36).

Complexo de Carney é uma síndrome caracterizada por tumores cardíacos, endócrinos, cutâneos e mixomas, assim como lesões pigmentadas na pele e mucosa. A displasia adrenal primária e pigmentada (PPNAD), principal característica do CC, é uma causa rara de síndrome de Cushing ACTH-independente que ocorre predominantemente em crianças e adultos jovens. Por meio de estudos de linkage genético, os cromossomos 17 q23-q24 e 2 pl 6 foram associados ao CC. Em cerca de $60 \%$ dos pacientes com CC, uma mutação foi identi- 
ficada no gene da proteína kinase dependente de cAMP (PRKARlA), principal mediador do sistema cAMP, diretamente envolvido na via de sinalização de ACTH. Mutações no gene PRKARIA, localizado no 17q2224, foram identificadas em adenomas adrenais, porém não em carcinomas adrenais; por outro lado, LOH dessa região foi encontrada em $23 \%$ dos adenomas e em $53 \%$ dos carcinomas adrenocorticais $(37,38)$.

Outros genes envolvidos na via de sinalização de ACTH foram previamente estudados, o do gene que codifica a subunidade $\alpha$ da proteína $\mathrm{G}$ estimulatória (GNAS) e o gene do receptor do ACTH (ACTH-R), da superfamília de receptores acoplados à proteína $\mathrm{G}$. A síndrome de McCune Albright, causada por mutações somáticas ativadoras do GNAS, é caracterizada por manifestações clássicas: a tríade displasia poliostótica fibrosa, manchas café-com-leite irregulares e disfunções endócrinas, como puberdade precoce, hipertireoidismo, gigantismo e síndrome de Cushing. Mutações no gene GNAS foram descritas em adenomas e hiperplasia macronodular adrenocortical, porém não foram identificadas em carcinomas adrenocorticais (39). $\mathrm{LOH}$ do $A C T H-R$ foi observado em 2 de 4 carcinomas e não foi encontrado em 15 adenomas, sugerindo que $A C T H-R$ desempenha um papel na diferenciação celular. Estudo por Northern blot e hibridização in situ sugeriu que $A C T H-R$ está hiper-regulado em adenomas funcionantes e hiporregulado em adenomas não funcionantes $\mathrm{e}$ em carcinomas (40). Adicionalmente, mutações não foram observadas no $A C T H-R$ em TAC, indicando que essas alterações não parecem ser comuns em TAC (41).

A síndrome de Beckwith-Wiedemann é caracterizada por macroglossia e gigantismo em neonatos e risco aumentado de tumores na infância, como tumor de Wilms, TCA, neuroblastoma e hepatoblastoma. Nesta síndrome, os genes CDKNIC, um inibidor IC da família das ciclinas dependentes de quinase com imprint paterno, e H19, um gene que não codifica proteína e atua como supressor tumoral com imprint materno, e o gene IGF2, todos os três localizados na região $11 \mathrm{pl5.5}$, parecem estar envolvidos na sua patogênese dessa síndrome (42). Além disso, LOH do locus 11 pl5.5 tem sido demonstrada em $67 \%$ dos carcinomas e $13 \%$ dos adenomas e parece ser responsável pela hiperexpressão de IGF2 encontrada em TAC (29). A expressão de H19 está elevada na adrenal fetal humana e diminui em cerca de $50 \%$ na adulta. Ainda, sua expressão está significativamente reduzida em CAC esporádicos não funcionantes e funcionantes, especialmente nos secretores de cortisol e aldosterona (43). O produto do gene CDKNICé encontrado em altos níveis na maioria dos tecidos humanos normais e parece regular proliferação celular, saída do ciclo celular e manutenção das células diferenciadas. A regulação da expressão de CDKNIC e HI9 é ACTH-dependente (43). Há também a perda de atividade da proteína codificada pelo CDKNIC em adenomas e carcinomas adrenocorticais virilizantes (44), sugerindo que este gene tem um papel fundamental na diferenciação adrenocortical. Em TAC esporádicos, mutações no CDKNIC não foram encontradas (45).

A síndrome de Li-Fraumeni é uma doença autossômica dominante descrita por Li e Fraumeni, em 1969. Caracteriza-se por múltiplos tumores nos indivíduos afetados, como sarcomas, câncer de mama, leucemia, tumores no sistema nervoso e TCA, os quais se iniciam precocemente. Essa síndrome familiar parece estar associada principalmente a mutações germinativas no gene TP53 em 70\% dos pacientes (46). Diversas mutações neste gene, incluindo as mutações p.R342X, p.R342P e p.R337G, também foram encontradas em crianças com TAC associadas à síndrome de Li-Fraumeni (47).

O gene NR5A2 codifica o fator esteroidogênico 1 (SFl), que tem papel essencial na regulação do desenvolvimento da adrenal e gônadas. O número de cópias desse gene parece estar aumentado na maioria dos TAC pediátricos. A análise por meio de imunoistoquímica demonstrou expressão aumentada da proteína SFl (31). Além disso, em adultos, tumores não esteroidogênicos não expressaram a proteína SFl (48). O aumento da expressão de NR5A2 e o aumento no número de cópias desse gene parecem ser mais comuns em TAC de crianças que em adultos (49). Nenhuma correlação foi encontrada entre o aumento da expressão de SFI e variáveis histológicas e/ou manifestação clínica da doença em crianças (31). No entanto, em pacientes adultos, a forte expressão de SFl correlacionou-se com pior prognóstico (48).

Os TAC estão associados à expressão elevada de membros da família de receptores de fator de crescimento epidérmico (EGFR), de fator de crescimento de fibroblasto (FGF-2), fator de crescimento transformador- $\beta$ (TGF- $\beta$ ), fator de crescimento transformador- $\beta$ l (TGF- $\beta 1)$, fator de crescimento endotelial vascular (VEGF) e interleucinas (34). O FGF-2 encontra-se hiperexpresso em tecidos adrenais, sendo um dos mais potentes mitógenos em células de cultura adrenal fetal e adulta, regulando a expressão do fator de crescimento semelhante à insulina do tipo 2 (IGF2). 
O potencial angiogênico dos TAC foi avaliado pelo estudo da expressão do VEGF que, em carcinomas, estava hiperexpresso em comparação aos adenomas. Além disso, houve diminuição dos níveis de VEGF após 1 mês de exérese de carcinomas, porém seu uso como marcador tumoral ainda precisa ser mais bem investigado (50). A expressão de TGF- $\beta 1$, que inibe a proliferação e regula o crescimento de células adrenais, apresentou-se reduzida em ACC, sem alterações na expressão ou presença de mutação em seu receptor $(51,52)$.

A expressão aumentada de $\operatorname{IGF2}(22,24,29,53)$ tem sido observada em TAC. O sistema IGF tem um papel importante no desenvolvimento e crescimento fisiológico da glândula adrenal (24). O IGF2 exerce efeitos mitogênicos através da interação com o receptor de $I G F I$ (IGFIR), e a expressão aumentada do IGF2/IGFIR pode desencadear uma série de eventos moleculares que podem levar à malignidade (25). A expressão aumentada do IGF2 tem sido encontrada na maioria dos adenomas pediátricos e dos carcinomas de crianças e adultos esporádicos $(22,29)$. Expressão de IGFIR aumentada parece ser um biomarcador de carcinoma adrenocortical em crianças, com potencial terapêutico, uma vez que um inibidor do IGF1R apresentou ação antitumoral em linhagens celulares de TACs de adulto e crianças (22).

O gene TP53 codifica uma importante proteína supressora tumoral, a P53, capaz de interromper o ciclo celular e induzir apoptose quando há dano ao DNA. Portanto, a perda da atividade da P53 pode promover ou facilitar o desenvolvimento do câncer (54). Mutações neste gene são encontradas em diversos tipos de tumores, inclusive TAC $(3,55)$. Nas regiões Sul e Sudeste do Brasil, em torno de $75 \%$ a $90 \%$ das crianças e $20 \%$ a $30 \%$ dos adultos com TAC apresentam a mutação germinativa P53 p.Arg337His em heterozigose $(3,4,55,56)$. Essa mutação leva à instabilidade da proteína na dependência do $\mathrm{pH}$ tecidual elevado, como encontrado nas adrenais (57). A análise de haplótipos deste mutante demonstrou um possível efeito fundador (58). Resultados de estudos recentes demonstraram que as famílias brasileiras carreando esse alelo mutante habitam locais ao longo da principal rota utilizada pelos comerciantes portugueses nos séculos XVIII e XIX (59). Em crianças, onde a mutação P53 p.R337H foi mais frequente, não houve associação direta entre a presença dessa mutação, o grau de malignidade ou $o$ prognóstico desses pacientes $(3,4,55)$. Em adultos, no entanto, essa e outras mutações do gene TP53 parecem associar-se a prognóstico mais desfavorável $(4,60)$.
Outra via envolvida na patogênese dos TAC é a via Wnt, que se caracteriza por um grupo de fatores de crescimento altamente conservados que atuam nos processos de homeostase e desenvolvimento (61). O evento principal da sinalização do Wnt é o acúmulo de $\beta$-catenina no citoplasma com consequente translocação ao núcleo e transcrição de genes alvo. Esse acúmulo pode levar a crescimento e proliferação celular descontrolados por desregulação da Via Wnt/ $\beta$-catenina (61). A via do Wnt tem sido implicada na patogênese de vários tipos de câncer $(62,63)$. A ativação da via $\mathrm{Wnt} / \beta$ catenina tem sido encontrada em hiperplasias, adenomas e carcinomas da adrenal (63), e mutações no gene CTNNB1, que codifica a $\beta$-catenina, têm sido identificadas em TCA. Alterações da via Wnt/ $\beta$-catenina parecem estar associadas a pior prognóstico em tumores adrenocorticais de adultos (63). Recentemente, dados do nosso laboratório demonstraram mutações do gene CTNNBI em 6\% dos TAC pediátricos, todas envolvendo sítios de fosforilação e degradação da $\beta$-catenina (64), em contraponto com a presença de mutações em $36 \%$ a $55 \%$ dos TAC de adultos, predominantemente em TAC não secretores $(63,65)$. Um achado interessante do nosso estudo é que todos os tumores apresentando mutação no gene CTNNBI também carreavam a mutação P53 p.R337H. Esse achado indica que, em TAC pediátricos, mutações dos genes CTNNBI e TP53 não são mutuamente exclusivas, como foi sugerido recentemente em TAC de adultos, carreando outras mutações do gene TP53 (63). Em análise por imuno-histoquímica, foi demonstrado acúmulo de $\beta$-catenina mesmo em tumores sem mutação no CTNNBI e, por qPCR, um aumento da expressão de CTNNBI e redução na expressão de seus inibidores (DKK3, SFRPI e $A X I N 1)$, sugerindo mecanismo de ativação da via pela perda de seus inibidores.

miRNAs, uma nova classe de pequenos RNAs reguladores da expressão gênica, foram avaliados em TAC de adultos por meio de uma análise de bioinformática integrada de transcriptoma para identificar o envolvimento destes na tumorigênese hipofisária (66). Seis miRNAs estavam alterados nos carcinomas em relação aos adenomas: miR-184, miR-210 e miR-503 estavam hiperexpressos, enquanto miR-511, miR-375 e miR215 , hipoexpressos, o que sugere papel diferencial na patogênese diferencial e agressividades. Outro estudo recente com TAC em adulto demonstrou 23 miRNAs diferentemente expressos ao comparar adenomas e carcinomas, sendo que a hipoexpressão do miR-195 e hi- 
perexpressão do miR-483 em carcinomas foi marcador de menor sobrevida (67). Também foi demonstrado papel funcional do miR-483 ao inibir o gene pró-apoptógeno PUMA, favorecendo seu papel na tumorigênese adrenal (68). Mais recentemente, foi avaliada a expressão de miRNA em tumores adrenocorticais pediátricos: miRs-99a e -100 são diferencialmente expressos e podem regular a via do mTOR (alvo da rapamicina em mamífero), o que sugere papel da via e destes miRNAs na tumorigênese adrenal (69).

Métodos mais modernos de análise da expressão gênica em larga escala foram desenvolvidos ao longo da última década. Onze recentes estudos analisaram a expressão gênica utilizando esses métodos em tumores adrenocorticais, sendo a maioria de adultos e, em apenas um deles, de crianças (70). Em conjunto, esses estudos demonstram diferença na expressão gênica comparando adenomas com carcinomas. Em carcinomas, são hiperexpressos genes da via do IGF2 (30,7173) e genes do ciclo celular como CCNE1, CCNE2, $M D M 2$, CDK2 e CDK4 (74), enquanto genes da esteroidogênese (MC2R, CYPIIB1, HSD3B2 e AKR IB1) são hipoexpressos (72). A via Wnt/Beta-Catenina também se encontra hiperexpressa em ambos adenomas e carcinomas de adrenal, mas, neste último grupo, de forma mais importante $(71,72,75)$. Em carcinomas, esses estudos também demonstraram que a expressão global gênica possibilitou separar os pacientes em grupos com diferentes prognósticos. A diferença na sobrevida e no estadiamento entre esses grupos de carcinoma leva ao questionamento se ACC são subgrupos diferentes ou apenas ACC em estágios diferentes. A análise de sobrevida e o perfil de transcriptoma diferentes sugerem a existência de subtipos distintos de ACCs $(71,72)$.

A procura por novos marcadores moleculares tem sido realizada por meio dessas técnicas de larga escala, como técnica de microarranjos, análise seriada da expressão gênica (SAGE), sequenciadores em larga escala de nova geração, entre outras, as quais permitem a análise simultânea de milhares de genes. Comparando-se a expressão gênica em adenomas e carcinomas, é possível identificar genes diferencialmente expressos nesses tecidos e compará-los ao tecido adrenal normal. Estudos utilizando essas técnicas estão sendo conduzidos em TACs de crianças e adultos. Devido à baixa incidência de carcinomas, os estudos têm incluído pequeno número de amostras e isso explica, em parte, a disparidade de genes encontrados. Adicionalmente, as diferentes plataformas de métodos de análise de larga escala, softwares e algoritmos usados na análise dos dados influenciam na variabilidade dos genes diferencialmente expressos.

Apesar das recentes descobertas e dos avanços tecnológicos na área, a patogênese molecular dos TCAs ainda é pouco esclarecida. Devemos ressaltar a importância de uma crescente quantidade de estudos na área, pois maiores descobertas levarão a um maior entendimento sobre a doença e sua origem, possibilitando maior chance de cura para pacientes acometidos por TCA. Dessa forma, esses estudos de expressão gênica de larga escala poderão facilitar o desenvolvimento de drogas para tratar alterações de acordo com as vias de sinalização específicas que estiverem alteradas.

Declaração: os autores declaram não haver conflitos de interesse científico neste estudo.

\section{REFERENCES}

1. Sandrini R, Ribeiro RC, DeLacerda L. Childhood adrenocortical tumors. J Clin Endocrinol Metab. 1997;82(7):2027-31.

2. Fassnacht M, Libe R, Kroiss M, Allolio B. Adrenocortical carcinoma: a clinician's update. Nat Rev Endocrinol. 2011;7(6):323-35.

3. Ribeiro RC, Sandrini F, Figueiredo B, Zambetti GP, Michalkiewicz E, Lafferty AR, et al. An inherited p53 mutation that contributes in a tissue-specific manner to pediatric adrenal cortical carcinoma. Proc Natl Acad Sci U S A. 2001;98(16):9330-5.

4. Latronico AC, Pinto EM, Domenice S, Fragoso MC, Martin RM, Zerbini MC, et al. An inherited mutation outside the highly conserved DNA-binding domain of the p53 tumor suppressor protein in children and adults with sporadic adrenocortical tumors. J Clin Endocrinol Metab. 2001;86(10):4970-3.

5. Soon PS, McDonald KL, Robinson BG, Sidhu SB. Molecular markers and the pathogenesis of adrenocortical cancer. Oncologist. 2008;13(5):548-61.

6. Allolio B, Fassnacht M. Clinical review: Adrenocortical carcinoma: clinical update. J Clin Endocrinol Metab. 2006;91(6):2027-37.

7. Ross NS, Aron DC. Hormonal evaluation of the patient with an incidentally discovered adrenal mass. $N$ Engl J Med. 1990;323(20):1401-5.

8. Arlt W, Biehl M, Taylor AE, Hahner S, Libe R, Hughes BA, et al. Urine steroid metabolomics as a biomarker tool for detecting malignancy in adrenal tumors. J Clin Endocrinol Metab. 2011;96(12):3775-84. Epub 2011 Sep 14.

9. Boland GW, Dwamena BA, Jagtiani Sangwaiya M, Goehler AG, Blake MA, Hahn PF, et al. Characterization of adrenal masses by using FDG PET: a systematic review and meta-analysis of diagnostic test performance. Radiology. 2011;259(1):117-26.

10. Lee MJ, Hahn PF, Papanicolaou N, Egglin TK, Saini S, Mueller PR, et al. Benign and malignant adrenal masses: CT distinction with attenuation coefficients, size, and observer analysis. Radiology. 1991;179(2):415-8.

11. Hamrahian $A H$, loachimescu AG, Remer EM, Motta-Ramirez G, Bogabathina $H$, Levin $\mathrm{HS}$, et al. Clinical utility of noncontrast computed tomography attenuation value (hounsfield units) to differentiate adrenal adenomas/hyperplasias from nonadenomas: Cleveland Clinic experience. J Clin Endocrinol Metab. 2005;90(2):871-7.

12. Ilias I, Sahdev A, Reznek RH, Grossman AB, Pacak K. The optimal imaging of adrenal tumours: a comparison of different methods. Endocr Relat Cancer. 2007;14(3):587-99. 
13. Macfarlane DA. Cancer of the adrenal cortex; the natural history, prognosis and treatment in a study of fifty-five cases. Ann R Coll Surg Engl. 1958;23(3):155-86.

14. Sullivan M, Boileau M, Hodges CV. Adrenal cortical carcinoma. J Urol. 1978;120(6):660-5.

15. Weiss LM, Medeiros LJ, Vickery AL Jr. Pathologic features of prognostic significance in adrenocortical carcinoma. Am J Surg Pathol. 1989;13(3):202-6.

16. Mendonca BB, Lucon AM, Menezes CA, Saldanha LB, Latronico $A C$, Zerbini $C$, et al. Clinical, hormonal and pathological findings in a comparative study of adrenocortical neoplasms in childhood and adulthood. J Urol. 1995;154(6):2004-9.

17. Latronico AC, Chrousos GP. Extensive personal experience: adrenocortical tumors. J Clin Endocrinol Metab. 1997;82(5):1317-24.

18. Michalkiewicz E, Sandrini R, Figueiredo B, Miranda EC, Caran E, Oliveira-Filho AG, et al. Clinical and outcome characteristics of children with adrenocortical tumors: a report from the International Pediatric Adrenocortical Tumor Registry. J Clin Oncol. 2004;22(5):838-45.

19. Luton JP, Cerdas S, Billaud L, Thomas G, Guilhaume B, Bertagna $X$, et al. Clinical features of adrenocortical carcinoma, prognostic factors, and the effect of mitotane therapy. N Engl J Med. 1990;322(17):1195-201.

20. Berruti A, Terzolo M, Sperone P, Pia A, Casa SD, Gross DJ, et al. Etoposide, doxorubicin and cisplatin plus mitotane in the treatment of advanced adrenocortical carcinoma: a large prospective phase II trial. Endocr Relat Cancer. 2005;12(3):657-66.

21. KhanTS, Imam H, Juhlin C, Skogseid B, Grondal S, Tibblin S, et al. Streptozocin and o, p'DDD in the treatment of adrenocortical cancer patients: long-term survival in its adjuvant use. Ann Oncol. 2000;11(10):1281-7.

22. Almeida MQ, Fragoso MC, Lotfi CF, Santos MG, Nishi MY, Costa $\mathrm{MH}$, et al. Expression of insulin-like growth factor-ll and its receptor in pediatric and adult adrenocortical tumors. J Clin Endocrinol Metab. 2008;93(9):3524-31.

23. Gicquel C, Boulle N, Logie A, Bourcigaux N, Gaston V, Le BoucY. [Involvement of the IGF system in the pathogenesis of adrenocortical tumors]. Ann Endocrinol (Paris). 2001;62(2):189-92.

24. Ilvesmaki V, Kahri Al, Miettinen PJ, Voutilainen R. Insulin-like growth factors (IGFs) and their receptors in adrenal tumors: high IGF-II expression in functional adrenocortical carcinomas. J Clin Endocrinol Metab. 1993;77(3):852-8.

25. Logie A, Boulle N, Gaston V, Perin L, Boudou P, Le Bouc Y, et al. Autocrine role of IGF-II in proliferation of human adrenocortical carcinoma NCl H295R cell line. J Mol Endocrinol. 1999;23(1):23-32.

26. GiordanoTJ. Adrenocortical tumors: an integrated clinical, pathologic, and molecular approach at the University of Michigan. Arch Pathol Lab Med. 2010;134(10):1440-3.

27. Kroiss $M$, Quinkler $M$, Lutz WK, Allolio $B$, Fassnacht $M$. Drug interactions with mitotane by induction of CYP3A4 metabolism in the clinical management of adrenocortical carcinoma. Clin Endocrinol (Oxf). 2011;75(5):585-91.

28. Gicquel C, Bertagna X, Gaston V, Coste J, Louvel A, Baudin E, et al. Molecular markers and long-term recurrences in a large cohort of patients with sporadic adrenocortical tumors. Cancer Res. 2001;61(18):6762-7.

29. Gicquel $C$, Bertagna $X$, Schneid $H$, Francillard-Leblond $M$, Luton $J P$, Girard F, et al. Rearrangements at the $11 \mathrm{p} 15$ locus and overexpression of insulin-like growth factor-II gene in sporadic adrenocortical tumors. J Clin Endocrinol Metab. 1994;78(6):1444-53.

30. Giordano TJ. Gene expression profiling of endocrine tumors using DNA microarrays: progress and promise. Endocr Pathol. 2003;14(2):107-16.

31. Pianovski MA, Cavalli LR, Figueiredo BC, Santos SC, Doghman $\mathrm{M}$, Ribeiro RC, et al. SF-1 overexpression in childhood adrenocortical tumours. Eur J Cancer. 2006;42(8):1040-3.
32. Gicquel C, Leblond-Francillard M, Bertagna X, Louvel A, Chapuis $\mathrm{Y}$, Luton JP, et al. Clonal analysis of human adrenocortical carcinomas and secreting adenomas. Clin Endocrinol (Oxf). 1994;40(4):465-77.

33. Sidhu S, Marsh DJ, Theodosopoulos G, Philips J, Bambach CP, Campbell $P$, et al. Comparative genomic hybridization analysis of adrenocortical tumors. J Clin Endocrinol Metab. 2002;87(7):3467-74.

34. Libe R, Fratticci A, Bertherat J. Adrenocortical cancer: pathophysiology and clinical management. Endocr Relat Cancer. 2007;14(1):13-28.

35. Chandrasekharappa SC, Guru SC, Manickam P, Olufemi SE, Collins FS, Emmert-Buck MR, et al. Positional cloning of the gene for multiple endocrine neoplasia-type 1. Science. 1997;276(5311):404-7.

36. Zwermann O, Beuschlein F, Mora P, Weber G, Allolio B, Reincke $M$. Multiple endocrine neoplasia type 1 gene expression is normal in sporadic adrenocortical tumors. Eur $\mathrm{J}$ Endocrinol. 2000;142(6):689-95.

37. Kirschner LS, Sandrini F, Monbo J, Lin JP, Carney JA, Stratakis CA. Genetic heterogeneity and spectrum of mutations of the PRKAR1A gene in patients with the carney complex. Hum Mol Genet. 2000;9(20):3037-46.

38. Bossis I, Stratakis CA. Minireview: PRKAR1A: normal and abnormal functions. Endocrinology. 2004;145(12):5452-8.

39. Fragoso MC, Domenice S, Latronico AC, Martin RM, Pereira MA, Zerbini MC, et al. Cushing's syndrome secondary to adrenocorticotropin-independent macronodular adrenocortical hyperplasia due to activating mutations of GNAS1 gene. J Clin Endocrinol Metab. 2003;88(5):2147-51.

40. Reincke M, Beuschlein F, Menig G, Hofmockel G, Arlt W, Lehmann R, et al. Localization and expression of adrenocorticotropic hormone receptor mRNA in normal and neoplastic human adrenal cortex. J Endocrinol. 1998;156(3):415-23.

41. Latronico AC. Role of ACTH receptor in adrenocortical tumor formation. Braz J Med Biol Res. 2000;33(10):1249-52.

42. Maher ER, ReikW. Beckwith-Wiedemann syndrome: imprinting in clusters revisited. J Clin Invest. 2000;105(3):247-52.

43. Liu J, Kahri Al, Heikkila P, Voutilainen R. Ribonucleic acid expression of the clustered imprinted genes, p57KIP2, insulin-like growth factor II, and H19, in adrenal tumors and cultured adrenal cells. J Clin Endocrinol Metab. 1997;82(6):1766-71.

44. El Wakil A, Doghman M, Latre De Late P, Zambetti GP, Figueiredo BC, Lalli E. Genetics and genomics of childhood adrenocortical tumors. Mol Cell Endocrinol. 2011;336(1-2):169-73.

45. Barzon L, Chilosi M, Fallo F, Martignoni G, Montagna L, Palu G, et al. Molecular analysis of CDKN1C and TP53 in sporadic adrenal tumors. Eur J Endocrinol. 2001;145(2):207-12.

46. Ruijs MW, Verhoef $S$, Rookus MA, Pruntel R, van der Hout AH, Hogervorst FB, et al.TP53 germline mutation testing in 180 families suspected of Li-Fraumeni syndrome: mutation detection rate and relative frequency of cancers in different familial phenotypes. J Med Genet. 2010;47(6):421-8.

47. Fiszer-Maliszewska L, Kazanowska B, Padzik J. p53 Tetramerization domain mutations: germline R342X and R342P, and somatic R337G identified in pediatric patients with Li-Fraumeni syndrome and a child with adrenocortical carcinoma. Fam Cancer. 2009;8(4):541-6.

48. Sbiera S, Schmull S, Assie G, Voelker HU, Kraus L, Beyer M, et al. High diagnostic and prognostic value of steroidogenic factor- 1 expression in adrenal tumors. $\mathrm{J}$ Clin Endocrinol Metab. 2010;95(10):E161-71.

49. Almeida MQ, Soares IC, Ribeiro TC, Fragoso MC, Marins LV, Wakamatsu $A$, et al. Steroidogenic factor 1 overexpression and gene amplification are more frequent in adrenocortical tumors from children than from adults. J Clin Endocrinol Metab. 2010;95(3):1458-62. 
50. Boulle N, Gicquel C, Logie A, Christol R, Feige JJ, Le Bouc Y. Fibroblast growth factor-2 inhibits the maturation of pro-insulin-like growth factor-II (Pro-IGF-II) and the expression of insulin-like growth factor binding protein-2 (IGFBP-2) in the human adrenocortical tumor cell line NCl-H295R. Endocrinology. 2000;141(9):3127-36.

51. Boccuzzi A, Terzolo M, Cappia S, De Giuli P, De Risi C, Leonardo $E$, et al. Different immunohistochemical patterns ofTGF-beta1 expression in benign and malignant adrenocortical tumours. Clin Endocrinol (Oxf). 1999;50(6):801-8.

52. Arnaldi G, Freddi S, Mancini T, Kola B, Mantero F. Transforming growth factor beta1: implications in adrenocortical tumorigenesis. Endocr Res. 2000;26(4):905-10.

53. Slater EP, Diehl SM, Langer P, Samans B, Ramaswamy A, ZieIke $A$, et al. Analysis by cDNA microarrays of gene expression patterns of human adrenocortical tumors. Eur $\mathrm{J}$ Endocrinol. 2006;154(4):587-98.

54. Soussi T. The history of p53. A perfect example of the drawbacks of scientific paradigms. EMBO Rep. 2010;11(11):822-6.

55. Sandrini F, Villani DP, Tucci S, Moreira AC, de Castro M, Elias LL. Inheritance of R337H p53 gene mutation in children with sporadic adrenocortical tumor. Horm Metab Res. 2005;37(4):231-5.

56. Figueiredo $B C$, Sandrini R, Zambetti GP, Pereira RM, Cheng C, Liu $W$, et al. Penetrance of adrenocortical tumours associated with the germlineTP53 R337H mutation. J Med Genet. 2006;43(1):91-6.

57. DiGiammarino EL, Lee AS, Cadwell C, Zhang W, Bothner B, Ribeiro $\mathrm{RC}$, et al. A novel mechanism of tumorigenesis involving $\mathrm{pH}$ -dependent destabilization of a mutant p53 tetramer. Nat Struct Biol. 2002;9(1):12-6.

58. Pinto EM, Billerbeck AE, Villares MC, Domenice S, Mendonca BB, Latronico AC. Founder effect for the highly prevalent R337H mutation of tumor suppressor p53 in Brazilian patients with adrenocortical tumors. Arq Bras Endocrinol Metabol. 2004;48(5):647-50.

59. Garritano S, Gemignani F, Palmero El, Olivier M, Martel-Planche G, Le Calvez-Kelm F, et al. Detailed haplotype analysis at the TP53 locus in p.R337H mutation carriers in the population of Southern Brazil: evidence for a founder effect. Hum Mutat. 2010;31(2):143-50.

60. Ragazzon B, Libe R, Gaujoux S, Assie G, Fratticci A, Launay P, et al. Transcriptome analysis reveals that $\mathrm{p} 53$ and \{beta\}-catenin alterations occur in a group of aggressive adrenocortical cancers. Cancer Res. 2010;70(21):8276-81.

61. Berthon A, Martinez A, Bertherat J, Val P. Wnt/beta-catenin signalling in adrenal physiology and tumour development. Mol Cell Endocrinol. 2011 Sep 10.

62. Polakis P.Wnt signaling and cancer. Genes Dev. 2000;14(15):1837-51.

63. Bonnet S, Gaujoux S, Launay P, Baudry C, Chokri I, Ragazzon $B$, et al. Wnt/\{beta\}-catenin pathway activation in adrenocortical adenomas is frequently due to somatic CTNNB1-activating mutations, which are associated with larger and nonsecreting tumors: a study in cortisol-secreting and -nonsecreting tumors. J Clin Endocrinol Metab. 2011;96(2):E419-26.

64. Leal LF, Mermejo LM, Ramalho LZ, Martinelli CE Jr, Yunes JA, Seidinger AL, et al. Wnt/\{beta\}-Catenin Pathway Deregulation in Childhood Adrenocortical Tumors. J Clin Endocrinol Metab. 2011;96(10):3106-14.

65. Mermejo LM, Leal LF, Colli LM, Martinelli CE, Moreira AC, Tone LG, et al., editors. P53 and CTNNB1 mutations reveal different patterns between pediatric and adult adrenocortical tumors. Endocr Rev. 2011;32:P1-39.

66. Tombol Z, Szabo PM, Molnar V, Wiener Z, Tolgyesi G, Horanyi J, et al. Integrative molecular bioinformatics study of human adrenocortical tumors: microRNA, tissue-specific target prediction, and pathway analysis. Endocr Relat Cancer. 2009;16(3):895-906.

67. Soon PS, Tacon LJ, Gill AJ, Bambach CP, Sywak MS, Campbell PR, et al. miR-195 and miR-483-5p Identified as Predictors of Poor Prognosis in Adrenocortical Cancer. Clin Cancer Res. 2009;15(24):7684-92.

68. Özata DM, Caramuta S, Velázquez-Fernández D, Akçakaya P, Xie H, Höög $A$, et al. The role of microRNA deregulation in the pathogenesis of adrenocortical carcinoma. Endocr Relat Cancer. Endocr Relat Cancer. 2011 Oct 27;18(6):643-55. Print 2011 Oct.

69. Doghman M, EI Wakil A, Cardinaud B, Thomas E, Wang J, Zhao W, et al. Regulation of insulin-like growth factor-mammalian target of rapamycin signaling by microRNA in childhood adrenocortical tumors. Cancer Res. 2010;70(11):4666-75.

70. Ragazzon B, Assie G, Bertherat J. Transcriptome analysis of adrenocortical cancers: from molecular classification to the identification of new treatments. Endocr Relat Cancer. 2011;18(2):R15-27.

71. Giordano TJ, Kuick R, Else T, Gauger PG, Vinco M, Bauersfeld J, et al. Molecular classification and prognostication of adrenocortical tumors by transcriptome profiling. Clin Cancer Res. 2009;15(2):668-76.

72. de Reynies A, Assie G, Rickman DS, Tissier F, Groussin L, Rene-Corail F, et al. Gene expression profiling reveals a new classification of adrenocortical tumors and identifies molecular predictors of malignancy and survival. J Clin Oncol. 2009;27(7):1108-15.

73. Soon PS, Gill AJ, Benn DE, Clarkson A, Robinson BG, McDonald $\mathrm{KL}$, et al. Microarray gene expression and immunohistochemistry analyses of adrenocortical tumors identify IGF2 and Ki-67 as useful in differentiating carcinomas from adenomas. Endocr Relat Cancer. 2009;16(2):573-83.

74. Szabo PM, Tamasi V, Molnar V, Andrasfalvy M, Tombol Z, Farkas R, et al. Meta-analysis of adrenocortical tumour genomics data: novel pathogenic pathways revealed. Oncogene. 2010;29(21):3163-72.

75. de Fraipont F, El Atifi M, Cherradi N, Le Moigne G, Defaye G, Houlgatte $R$, et al. Gene expression profiling of human adrenocortical tumors using complementary deoxyribonucleic Acid microarrays identifies several candidate genes as markers of malignancy. J Clin Endocrinol Metab. 2005;90(3):1819-29. 